\title{
Probabilistic Electrical Power Generation Modeling Using Genetic Algorithm
}

\author{
Ahmed S. Al-Abdulwahab \\ Electrical and Computer Engineering Department, College of Engineering \\ King Abdulaziz University \\ P.O.Box 80204, Jeddah 21589 \\ SAUDI ARABIA
}

\begin{abstract}
Generation system reliability assessment is an important task which can be performed using deterministic or probabilistic techniques. The probabilistic approaches have significant advantages over the deterministic methods. However, more complicated modeling is required by the probabilistic approaches. Power generation model is a basic requirement for this assessment. One form of the generation models is the well known capacity outage probability table (COPT). Different analytical techniques have been used to construct the COPT. These approaches require considerable mathematical modeling of the generating units. The units' models are combined to build the COPT which will add more burdens on the process of creating the COPT. This paper proposes the utilization of the Genetic Algorithm (GA) to sample the states of the COPT without engaging in analytical units modeling. The simple binary representation, " 0 " and " 1 " is used to model the states of generating units. The effect of the GA parameters is examined. The proposed technique is proven to be an effective approach to build the generation model. The proposed technique is applied to the RBTS.
\end{abstract}

\section{General Terms}

Modeling, Genetic algorithm application.

\section{Keywords}

Genetic algorithm, power system reliability, power generation modeling.

\section{INTRODUCTION}

Generating capacity adequacy assessment is an important aspect of power system planning. In order to maintain a desired level of reliability, the system must have a capacity reserve in excess of the actual load demand. Many techniques have been developed to determine the required level of capacity reserve in a system. These techniques can be divided into two types, probabilistic and deterministic [1-4]. A number of Canadian surveys have been conducted and it was concluded from these surveys that Canadian utility practice has moved over time from deterministic approaches to probabilistic techniques [5].

Two models are required to conduct probabilistic adequacy assessment of the system, the load model and the generation model. The most commonly used load models are the daily peak load variation curve (DPLVC), and the load duration curve (LDC). These two models are fairly straight forward to build. The generation model is usually presented in the form of a capacity outage probability table (COPT) which includes the available or unavailable capacity levels and their corresponding probabilities. The COPT has been created using different techniques [6-7]. These approaches can be categorized into two main categories analytical methods and Monte Carlo simulation (MCS) techniques .Analytical approaches require mathematical modeling for the generating units. The unit's models are then combined to generate the system states which will add more burdens on the process of creating the COPT. MCS does not require extensive mathematical modeling, however, a large number of simulation need to be considered to reach an acceptable confident level. Moreover, MCS is a random process in which system states can be repeated many times unnecessarily. The proposed technique utilizes the GA which does not require analytical representation to recover the system states which in turn formalize the COPT. GA is random yet controlled process. The appearance of previously generated system states can be controlled and reduced. GA has emerged as a powerful search tool used for search and optimization [8-14]. Ref. [10] utilized the GA to calculate the reliability indices of the system. Creating COPT was embedded in the reliability assessment process. This paper illustrates the utilization of GA to specifically create the COPT.

A typical GA starts with a random population of chromosomes encoded as strings of symbols. Then, it goes through a number of iterations in which the population at the beginning of each iteration is replaced by another population. The population produced at each step is called a generation. The chromosomes of each new generation are produced by the reproduction process. Reproduction of the new generation involves three operations applied to the current generation. These operations are selection, crossover and mutation.

Conventionally, a GA maximizes an objective function. This function measures the fitness of each chromosome. Many optimization techniques are based on identifying whether a solution is feasible or not. The infeasible solutions are rejected even though some of the rejected solutions may be good partial solutions. The GA can utilize the infeasible solutions by penalizing the solutions that breaks a constraint by reducing their fitness values.

This paper presents the application of the GA as a search technique to accumulate the most effective capacity states to build the COPT. Two major factors affect the accuracy of the COPT, the number of states and the probability of these states. These two factors are monitored in the analysis. The effect of the GA 
parameters is illustrated in this paper. The concepts presented are demonstrated by application to the RBTS.

\section{GA SEARCH TECHNIQUE}

The GA is used as a search tool to construct the generation model (COPT). The GA generates system states represented as chromosomes and the fit individuals are sampled to build the COPT. For a system with $\mathrm{N}$ generating units which can be either "in service" or "out of service", the total number of system states is $2 \mathrm{~N}$. The probabilities of these states should add up to 1.0. However, for large systems, it is not practical to include all of the system states in the COPT. Some of these states have low probability of occurrence which means that they are rare to occur. These states do not affect significantly the system reliability assessment. In fact, it is the usual practice to truncate these states out of the COPT [6]. The proposed application of the GA to build the COPT is designed to truncate the insignificant states and its process is summarized in the following steps:

1) An initial population is generated randomly. This population contains individual chromosomes. Each chromosome represents a system state which is encoded as a binary string with a length equal to the number of generating units. Each unit is represented by one bit with a value of ' 1 ' or ' 0 ' corresponding to 'in service' or 'out of service' states. The probability of the units to be found in these two states is given by Equation 1.

$$
\operatorname{prob}_{j}=\left\{\begin{array}{c}
1-F O R_{j} \text { if } \text { state }_{i j}=1 \\
F_{j} \text { if } \text { state }_{i j}=0
\end{array}\right.
$$

where,

stateij is the state of unit $\mathrm{j}$ in system state $\mathrm{i}$,

probj is the state probability of unit $\mathrm{j}$,

FOR $\mathrm{j}$ is the Forced Outage Rate of unit $\mathrm{j}$

2) The fitness of each system state (individual) is evaluated by calculating its probability. State probability is calculated using Equation 2. In a given population, there are some generated system states which are repeated. The fitness of these states is set to a very small value so that they are ignored and truncated from the COPT. The unrepeated states are added to a system states array. The system states array will be used later to construct the COPT.

$$
\text { State } \operatorname{prob}_{i}=\prod_{j=1}^{N} \text { prob }_{j}
$$

where,

$$
\mathrm{N} \text { is number of generating units }
$$

3) The "capacity in service" and the "capacity out of service" are calculated for each system state recovered in the system states array for the current population using Equations 3 and 4 respectively.

$$
\begin{aligned}
& \text { State Cap } \operatorname{in}_{i}(M W)=\sum_{j=1}^{N} \text { State }_{i j} * \text { Cap }_{j} \\
& \text { State Cap Out }(M W)=T I C-\text { State Cap in }
\end{aligned}
$$

where,

$$
\begin{aligned}
& \text { Capj is the capacity of unit } j \\
& \text { TIC is the Total Installed Capacity }
\end{aligned}
$$

4) Selection of fit strings (system states), to be used to generate the new generation is done using the tournament selection technique. The states that have higher probability have more chances to be selected. The population members are randomly divided into subgroups and members with the best fitness among the subgroups get selected for reproduction. This process decreases the chances of generating system states with low probability of occurrence. Therefore, the selection process acts as the truncation technique.

5) Crossover operation follows the selection process. The newly selected strings are paired together at random. Then, an integer position along every pair of strings is selected uniformly at random. Finally, based on a probability of crossover, the paired strings undergo crossing over at the integer position along the string. This results in new pairs of strings (system states).

6) Mutation operation follows the previous two GA operation to finalize the reproduction process. An occasional random alteration of a string based on probability of mutation is performed. This involves changing a 1 to a 0 and vice versa.

7) At this stage, the new generation is generated. To keep track of the progress in collecting the fit system states, the total probability of the collected states in the system states array is calculated. This probability is designated as "cumulative probability" and calculated using Equation 5. Then steps 2 to 7 are repeated.

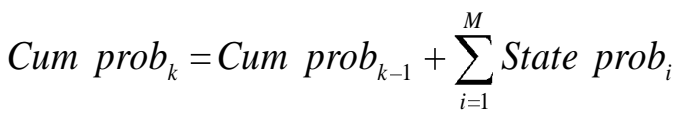

where,

$\mathrm{M}$ is the total number of states collected in the kth generation. Obviously, the Cum prob ${ }_{0-1}$ in the initial population is zero. The cumulative probability approaches 1.0 as reproduction iterations precede.

8) Different stopping criteria can be used. A maximum number of generations (iterations) can be used as a stopping criterion. Another stopping criterion can be used which is stopping the reproduction operation when there is no improvement (increase) in the cumulative probability of the collected states. Also, the regeneration can be terminated after finding the desired maximum cumulative probability. In this paper, the maximum number of generation is used as the stopping criterion.

The final COPT is constructed by arranging the recovered states in the system states array in a descending order using the capacity out of service. The states with equal capacities are replaced by a single state with the probability equals to the sum of the probabilities of these states.

\section{CASE STUDIES}

The technique described above is illustrated on a small system composed of three generating units with a total installed capacity (TIC) of $60 \mathrm{MW}$. The system data is shown in Table 1. 
Table 1. Test system data.

\begin{tabular}{|c|c|c|}
\hline & Capj (MW) & FORj \\
\hline Unit 1 & 10 & 0.02 \\
\hline Unit 2 & 30 & 0.04 \\
\hline Unit 3 & 20 & 0.10 \\
\hline
\end{tabular}

The total number of the possible system states for this system is 23 (8 states). Since this system is very small then all of the states will be recovered by the GA search. A population size of 10 is considered in this case. The initial population of the first generation which was generated randomly is shown in Table 2 .

As explained previously, each row in this population represents a system state which is encoded as a binary string with a length equal to the number of generating units ( 3 units). It can be seen from Table 2 that States 6 and 10 and States 3, 7 and 8 are identical states. The repeated states will be penalized in the next step. The state probability of each unit is calculated using Equation 1 utilizing the data given in Table 1 and the information in Table 2. Then system state probabilities of the initial population are calculated using Equation 2 and shown in Table 3.

Table 3 shows that the fitness values for the repeated states (States 3, 6 and 7) are assigned a very small value appears as zeros. The unrepeated states are added to system states array and their corresponding "in service" and "out of service" capacities are calculated using Equations 3 and 4 respectively. The cumulative probability of the recovered states is also calculated using Equation 5. The resulting system states array is shown in Table 4.

Table 2. Initial population for the test system.

\begin{tabular}{|c|c|c|c|}
\hline & Unit 1 & Unit 2 & Unit 3 \\
\hline System State & & State ij & \\
\hline 1 & 0 & 0 & 0 \\
\hline 2 & 0 & 0 & 1 \\
\hline 3 & 1 & 0 & 0 \\
\hline 4 & 1 & 1 & 0 \\
\hline 5 & 1 & 0 & 1 \\
\hline 6 & 0 & 1 & 1 \\
\hline 7 & 1 & 0 & 0 \\
\hline 8 & 1 & 0 & 0 \\
\hline 9 & 0 & 1 & 0 \\
\hline 10 & 0 & 1 & 1 \\
\hline
\end{tabular}

Table 3. State probability for the initial population.

\begin{tabular}{|c|c|}
\hline System State & State probi \\
\hline 1 & 0.00008 \\
\hline 2 & 0.00072 \\
\hline 3 & 0.00000 \\
\hline
\end{tabular}

\begin{tabular}{|l|l|}
\hline 4 & 0.09408 \\
\hline 5 & 0.03528 \\
\hline 6 & 0.00000 \\
\hline 7 & 0.00000 \\
\hline 8 & 0.00392 \\
\hline 9 & 0.00192 \\
\hline 10 & 0.01728 \\
\hline
\end{tabular}

It can be seen from Table 4 that the cumulative probability is very low which indicates that not all the significant states with high probabilities are recovered. A new population is produced by manipulating the current population using the GA operation (selection, crossover and mutation). The resulting new generation is shown in Table 5.

The new population shown in Table 5 revealed a new state which was not recovered in the previous population. The probability for this state is calculated using Equation 2 which yields a value of 0.84672 . The rest of the states were recovered previously and therefore, the probabilities for these states are set to a very small value. The new system array is updated and shown in Table 6.

Table 4. System states array for the first generation.

\begin{tabular}{|c|c|c|c|}
\hline $\begin{array}{c}\text { System } \\
\text { State }\end{array}$ & $\begin{array}{c}\text { State Cap Ini } \\
(\mathrm{MW})\end{array}$ & $\begin{array}{c}\text { State Cap } \\
\text { Outi (MW) }\end{array}$ & State Probi \\
\hline 1 & 0 & 60 & 0.00008 \\
\hline 2 & 20 & 40 & 0.00072 \\
\hline 3 & 40 & 20 & 0.09408 \\
\hline 4 & 30 & 30 & 0.03528 \\
\hline 5 & 10 & 50 & 0.00392 \\
\hline 6 & 30 & 30 & 0.00192 \\
\hline 7 & 50 & 10 & 0.01728 \\
\hline
\end{tabular}

Table 5. The new population of the second generation.

\begin{tabular}{|c|c|c|c|}
\hline System State & Unit 1 & Unit 2 & Unit 3 \\
\hline 1 & 1 & 1 & 0 \\
\hline 2 & 1 & 1 & 0 \\
\hline 3 & 1 & 1 & 0 \\
\hline 4 & 0 & 1 & 0 \\
\hline 5 & 1 & 1 & 0 \\
\hline 6 & 1 & 1 & 0 \\
\hline 7 & 1 & 0 & 1 \\
\hline 8 & 1 & 1 & 0 \\
\hline 9 & 1 & 1 & 1 \\
\hline 10 & 0 & 0 & 1 \\
\hline
\end{tabular}


Table 6. System states array after the second generation.

\begin{tabular}{|c|c|c|c|}
\hline $\begin{array}{c}\text { System } \\
\text { State }\end{array}$ & $\begin{array}{c}\text { State Cap Ini } \\
(\mathrm{MW})\end{array}$ & $\begin{array}{c}\text { State Cap } \\
\text { Outi (MW) }\end{array}$ & State Probi \\
\hline 1 & 0 & 60 & 0.00008 \\
\hline 2 & 20 & 40 & 0.00072 \\
\hline 3 & 40 & 20 & 0.09408 \\
\hline 4 & 30 & 30 & 0.03528 \\
\hline 5 & 10 & 50 & 0.00392 \\
\hline 6 & 30 & 30 & 0.00192 \\
\hline 7 & 50 & 10 & 0.01728 \\
\hline 8 & 60 & 0 & 0.84672 \\
\hline
\end{tabular}

It can be seen from Table 6 that all of the system states (23) are recovered after the second generation. This is due to the size of the test system which is very small. The final step is to rearrange the states and merge the states with same capacity level (States 4 and 6). Table 7 shows the final COPT.

The final COPT shown in Table 7 obtained for the three-units test system was verified and compared with the results obtained for the same system using the conventional recursive algorithm presented in Reference [6]. This comparison yields identical results.

Table 7. The final COPT for the test system.

\begin{tabular}{|c|c|c|}
\hline $\begin{array}{c}\text { State Cap Outi } \\
(\mathrm{MW})\end{array}$ & $\begin{array}{c}\text { State Cap Ini } \\
(\mathrm{MW})\end{array}$ & State Probi \\
\hline 60 & 0 & 0.00008 \\
\hline 50 & 10 & 0.00392 \\
\hline 40 & 20 & 0.00072 \\
\hline 30 & 30 & 0.03720 \\
\hline 20 & 40 & 0.09408 \\
\hline 10 & 50 & 0.01728 \\
\hline 0 & 60 & 0.84672 \\
\hline
\end{tabular}

The COPT was built without actually modeling the generating units. The " 0 " and " 1 " are used to model the states of the units. Moreover, the significant capacity states were recovered by the GA search.

The developed technique is applied to the RBTS [15]. The RBTS is an educational test system that has 11 generating units, ranged from $5 \mathrm{MW}$ to $40 \mathrm{MW}$. The total number of states for the RBTS is 211 (2048) states. The GA search method is applied to the RBTS with 10 generations (iterations) and a population size of 300 . The result of this simulation is shown in Figure. 1.

It can be seen from Figure 1 that both the number of states and the cumulative probability increase in every generation. The GA search technique accumulated around 490 states in ten generations which represents $24 \%$ of the total number of states (2048). The cumulative probability of these states represents $99.9788 \%$ of the total probability for all states which is $100 \%$. More accurate result can be achieved by increasing the population size and number of generation if desired. The effect of the GA parameters is examined in the next section.

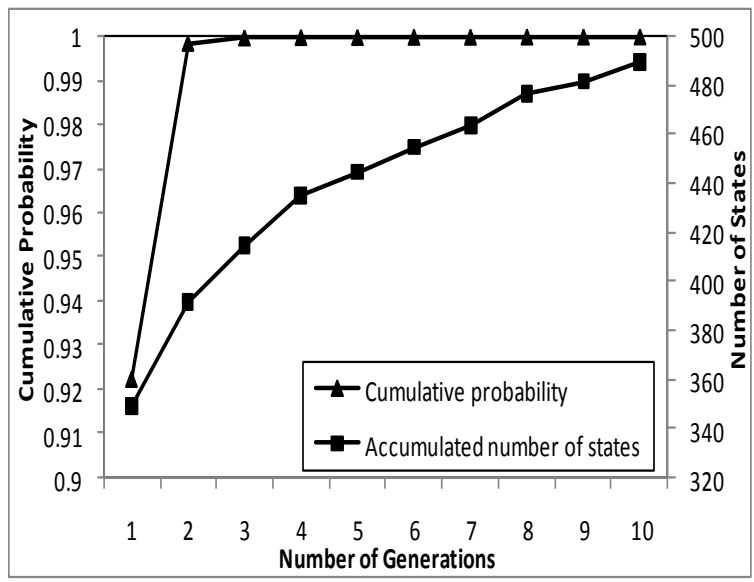

Figure 1. GA search result for the RBTS

\section{SENSITIVITY ANALYSIS}

The effect of the GA parameters on the generation model is examined using the RBTS. These parameters include the generation size, population size and crossover probability. Two characteristics of the COPT are tracked, the number of states included in the COPT and the total cumulative probability of these states.

The effect of number of generations is considered. A population size of 100 is used in this case. Number of generations of 10, 20, $30,40,50$ and 100 are examined. Figure 2 shows the number of states recovered for the different number of generations. The cumulative probability of the recovered states is also shown for the corresponding number of generation.

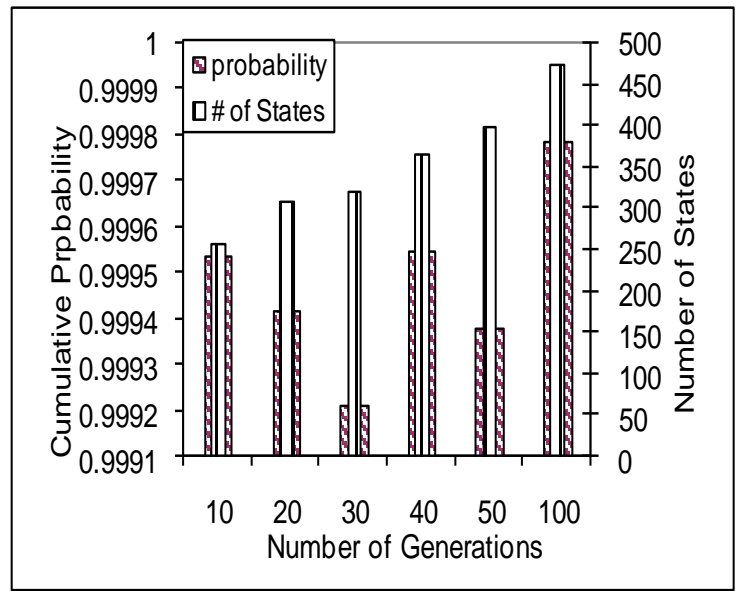

Figure 2. Number of states and the corresponding cumulative probability for different number of generations and a population size of 100 . 
It can be seen from Figure 2 that the number of states recovered in the COPT increases consistently with the increase in the number of generation. However, the cumulative probability of the recovered states fluctuates with the increase in the number of generation. Two factors can cause this phenomenon, either the number of generations or the population size of 100 is not large enough for the GA operations to recover all the effective states. A higher population size of 300 is considered and the result is shown in Figure 3.

It can be seen from Figure 3 that the number of the recovered states and their corresponding cumulative probabilities increase consistently with the increase in the number of generation. This reflects that no significant states were not recovered with the population size of 300 .

Figure 3 shows that a noticeable increase in the cumulative probability was associated with the increase in the number of generation from 20 to 30 . Nevertheless, the increase in the cumulative probability achieved with the increase in the number of generation from 50 to 100 was not significant. Therefore, a high enough number of generations need to be considered in order to have confident in the resulting COPT.

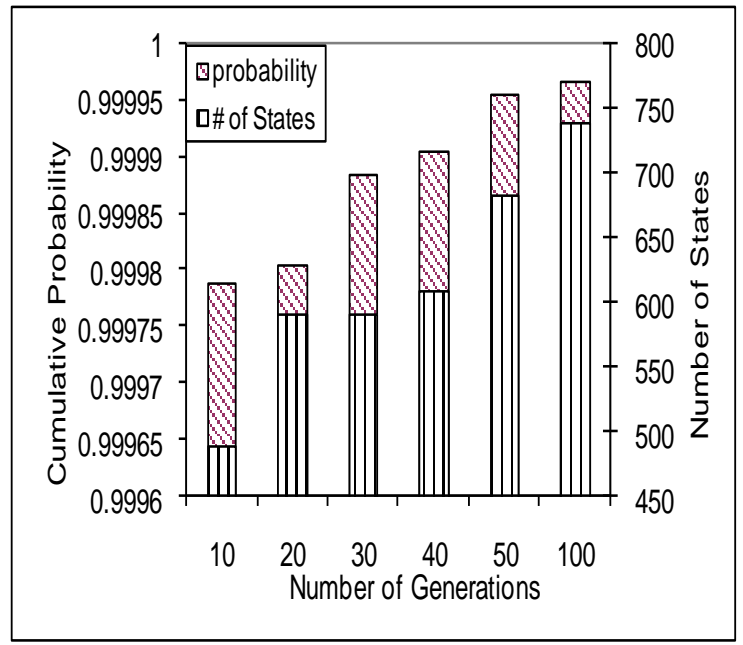

Figure 3. Number of states and the corresponding cumulative probability for different number of generations and a population size of 300 .

The effect of the population size is considered next. A number of generations of 50 is used in this analysis. Different population sizes ranges from 50 to 1000 are considered. Figure 4 shows the number of states recovered with the different population sizes. The cumulative probability of the recovered states is also shown for the corresponding population size.

It can be seen from Figure 4 that the number of states consistently increases with the increase in the population size. However, the cumulative probabilities fluctuate with this increase in the population size. This fluctuation is due to the fact that some of the effective states with relatively high probability were not recovered with the relatively small number of generation considered. The cumulative probability started to settle at the population size of 500 .

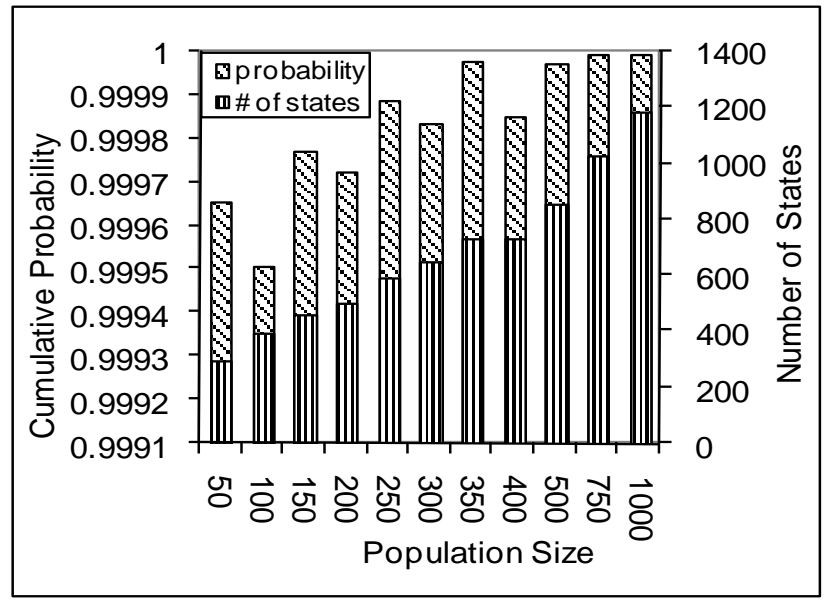

Figure 4. Number of states and the corresponding cumulative probabilities for different population sizes and a number of generations of 50.

A larger number of generations of 100 is considered and the result is shown in Figure 5. It can be seen from this figure that the number of states recovered and the corresponding cumulative probabilities are consistently increasing with the increase in the population size.

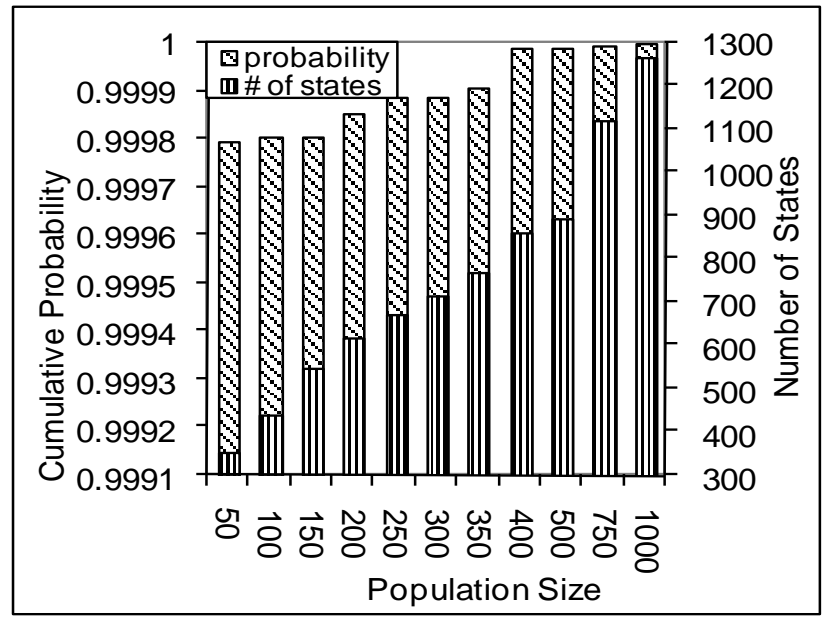

Figure 5. Number of states and the corresponding cumulative probabilities for different population sizes and a number of generations of 100 .

It can be seen from Figure 5 that there is a significant increase in the number of states when the population size increased from 500 to 750 . Nevertheless, very limited increase in the cumulative probability occurred in this case. Therefore, it is not needed to go beyond the population size of 500 in order to achieve an acceptable level of confident.

In fact, it can be seen from Figure 4 and Figure 5 with a population size of 500, that similar number of states and similar cumulative probability were recovered in both cases. Therefore, there is no need to go for a larger number of generations with a large population size to reach a similar confident level. 
The effect of the crossover probability is considered. A number of generations of 100 and a population size of 400 are used in this analysis. Different crossover probabilities ranges from 0.005 to 0.5 are considered. Figure 6 shows the number of states recovered for the different crossover probabilities. The cumulative probability of the recovered states is also shown for the corresponding crossover probabilities.

Figure 6 shows that the number of the recovered states and the corresponding cumulative probabilities fluctuate with the increase in the crossover probability. However, the range of these fluctuating is relatively small compared to that with the other GA parameters as illustrated in the previous group of figures. Therefore, the crossover probability does not affect the COPT construction significantly under these conditions.

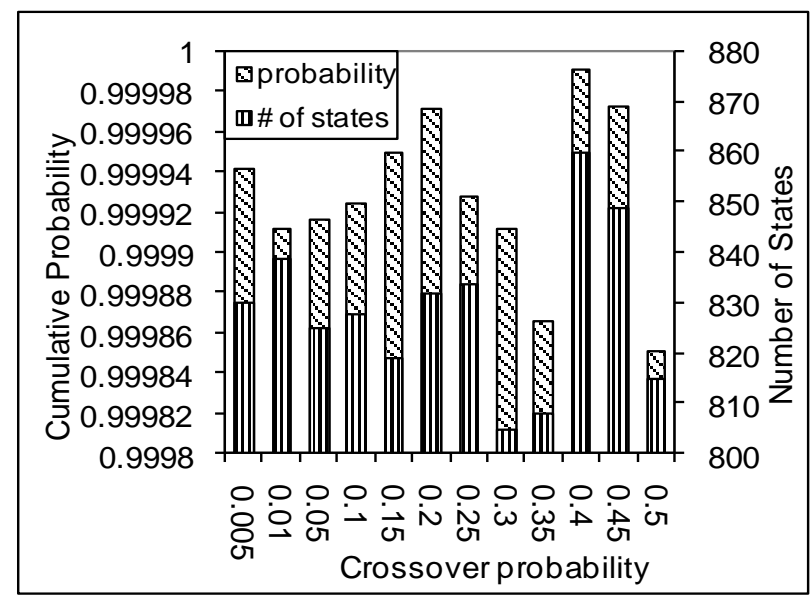

Figure 6. Number of states and the corresponding cumulative probabilities for different crossover probabilities and a number of generations of 100 and a population size of 400 .

\section{CONCLUSIONS}

This paper presented an effective method based on the GA search to create the power generation model. This model is one of the basic requirements for the generation power system reliability evaluation. The proposed method uses GA as a tool to search for the effective states that have high probability of occurrence. The proposed approach has two main features; first of all, no complicated modeling is required to represent the generating units. The binary representation, " 0 " and " 1 ", is used to represent the states of the generating units Second of all, it has the capability to truncate the ineffective capacity states and yet results with an accurate COPT. This feature is very useful when dealing with large systems.

The proposed technique was tested on the RBTS. It was found that the accuracy of the created generation model depends on the GA characteristics. Therefore, caution has to be taken when setting the values of the GA operators.

\section{REFERENCES}

[1] IEEE Committee Report, 1978. Bibliography on the Application of Probability Methods in Power System Reliability Evaluation, 1971-1977, IEEE Transactions on Power Apparatus and Systems, Vol. PAS-97, No. 6, pp. 2235-2242.

[2] Allan, R. N., Billinton, R. and Lee, S. H. 1984. Bibliography on the Application of Probability Methods in Power System
Reliability Evaluation, 1977-1982, IEEE Transactions on Power Apparatus and Systems, PAS-103, pp. 275-282, No. 2.

[3] Allan, R. N., Billinton, R., Shahidehpour, S. M. and Singh, C. 1988. Bibliography on the Application of Probability Methods in Power System Reliability Evaluation, 1982-87, IEEE Transactions on Power Apparatus and Systems, Vol. 3, No. 4, pp. $1555-1564$.

[4] Billinton, R., 1972. Bibliography on the Application of Probability Methods in Power System Reliability Evaluation, IEEE Transactions on Power Apparatus and Systems, Vol. 91, No. 2, pp.649-660.

[5] Billinton, R. 1991. Criteria Used by Canadian Utilities in the Planning and Operation of Generating Capacity, Applied Reliability Assessment in Electric Power Systems, IEEE press, New York, pp. 186-191.

[6] Allan, R. N., and Billinton, R., 1996. Reliability Evaluation of Power Systems, Plenum Press, New York.

[7] Singh, C. and Gubbala, N. V. 1996. Comparison of unit addition and fast Fourier transform methods for generation system reliability evaluation, Electrical Power \& Energy Systems Vol. 18 No. 4, pp. 203-205.

[8] Abdulwhab, A. and Billinton, R. 2004. Maintenance Scheduling Using a Genetic Algorithm (GA) with a probabilistic Fitness Function, Journal of Electric Power Components and Systems, Volume 32, Number 12, pp. 12391254 (16).

[1] Goldberg, D. E. 1989. Genetic Algorithms in Search, Optimization and Machine learning, Addison-Wesley Publishing company.

[9] Samaan, N. and Singh, C. 2001. Using of Genetic Algorithms to Evaluate Frequency and Duration Indices for Generation System Reliability, in Proc. ISAP 2001 Intelligent System Application to Power System Conf., Budapest, Hungry, pp. 251-256.

[10] Masoum, M. A., Ladjevardi, S. M., Jafarian, A. and Fuchs, E. F. 2004. Optimal Placement, Replacement and Sizing of Capacitor Banks in Distorted Distribution Networks by Genetic Algorithms, IEEE transactions on power delivery, vol. 19 , no. 4.

[11] Todorovski, M. and Dragoslav, 2003. A Power Flow Method Suitable For Solving OPF Problems Using Genetic Algorithms, EUROCON 2003 Ljubljana, Slovenia.

[12] Li Hui, Han Li, He Bei, and Yang Shunchang, 2001. Application Research Based On Improved Genetic Algorithm For Optimum Design Of Power Transformers, Electrical Machines and Systems, 2001, ICEMS 2001, Proceedings of the Fifth International Conference on Volume 1, 18-20 Page(s):242 - 245 vol.1.

[13] Rivas-Davalos, F. and Irving, M. R. 2003. An Efficient Genetic Algorithm For Optimal Large-Scale Power Distribution Network Planning, Paper accepted for presentation at 2003 IEEE Bologna Power Tech Conference, June 23-26, Bologna, Italy.

[14] Billinton, R., Kumar, S., Chowdhury, K., Chu, K., Debnath, K., Goel, L., Khan, E., Kos, P., Nourbakhsh, G., and OtengAdjei, J. 1989. A Reliability Test System for Educational Purposes - Basic Data, IEEE Transactions on Power Systems, Vol. 4, No. 3, pp. 1238-1244. 